\title{
Studies on the mid-term effects of the double-modulation CPT clock
}

A. Bouvier, C. Calosso, P. Yun, E. de Clercq, S. Guerandel

A. Bouvier, C. Calosso, P. Yun, E. de Clercq, S. Guerandel, "Studies on the mid-term effects of the double-modulation CPT clock," Proc. SPIE 11852, International Conference on Space Optics — ICSO 2020, 1185247 (11 June 2021); doi: 10.1117/12.2599644

SPIE Event: International Conference on Space Optics - ICSO 2021, 2021, Online Only 


\section{International Conference on Space Optics-ICSO 2020}

Virtual Conference

30 March-2 April 2021

Edited by Bruno Cugny, Zoran Sodnik, and Nikos Karafolas
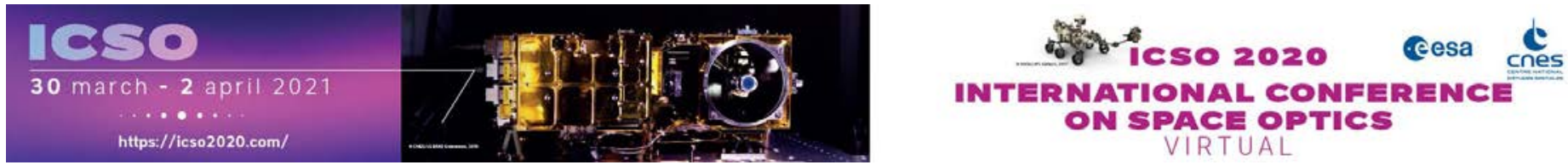

Studies on the mid-term effects of the Double Modulation CPT clock

\section{Cesa isonematang lecnes}




\title{
Studies on the mid-term effects of the Double Modulation CPT clock
}

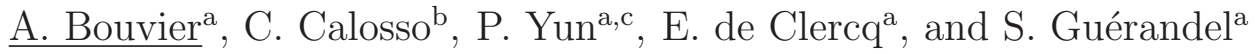 \\ ${ }^{a}$ LNE-SYRTE, Observatoire de Paris, Université PSL, CNRS, Sorbonne Universités , 61 av. de \\ l'Observatoire, 75014 Paris, France \\ bIstituto Nazionale di Ricera Metrologica, INRIM, Strada delle Cacce 91, Torino, Italy \\ 'Key Laboratory of Time and Frequency Primary Standards, National Time Service Center, \\ Chinese Academy of Sciences, 71060 Xi'an, China
}

\begin{abstract}
We present recent studies on a continuous compact atomic clock based on the double modulation coherent population trapping (DM-CPT) technique. A short term frequency stability of $2.9 \times 10^{-13} \tau^{-\underline{1}}$ up to $100 \mathrm{~s}$ averaging time is reported. A new technique and time sequence in order to gain in stability in the $1000 \mathrm{~s}$ is presented, allowing a frequency stability of $2.0 \times 10^{-13}$ at this averaging time, which is better by almost an order of magnitude than the previously published results.
\end{abstract}

Keywords: Compact atomic clock, CPT, Metrology

\section{INTRODUCTION}

Atomic clocks are precision devices delivering a very stable frequency across time. They are used for different purpose where there is a need for a precise time and frequency measurement. In particular, they are widely used in satellites, inertial navigation, data transfer, telecommunication synchronization, etc $[1,2]$.

In this paper, we describe the recent studies on a continuous compact atomic clock based on the double modulation coherent population trapping (DM-CPT) technique [3]. We modulate synchronously the phase and the polarisation of a bichromatic laser beam in order to trap more atoms in the so-called dark state, increasing the observed atomic signal. Results of a short term frequency stability of $2.9 \times 10^{-13} \tau^{-\frac{1}{2}}$ up to $100 \mathrm{~s}$ averaging time is reported. However, these are best selection results and a typical frequency stability shows an integration in $\tau^{-\frac{1}{2}}$ limited to $10 \mathrm{~s}$. In the reported noise contribution budget, we show that the main limitation is the stability of the microwave power used to generate the bichromatic laser. We propose a new method to lock the microwave power using the AC Stark shift effect and measure a frequency stability almost 10 time better after $1000 \mathrm{~s}$. These preliminary results are encouraging and demonstrate that the DM-CPT technique is well suited for the development of a high performance continuous atomic clock with a potential compact and robust design thanks to its in-line architecture. This clock could find application in telecommunications, instrumentation or global navigation satellite systems.

\section{EXPERIMENTAL SET-UP}

The experimental set up used for this paper is reported in the figure 1. It is a simple in line optical scheme. We modulate a Distributed FeedBack laser source at 4,6 GHz around the central frequency in order to generate a bichromatic laser field where each side-band is separated by $9,2 \mathrm{GHz}$ one from the other. The laser source is power and frequency locked. We then modulate synchronously the phase and the polarisation of our laser beam before the clock cell, hence the name double modulation (DM). The polarisation modulation keeps the atoms out of the extreme Zeeman sublevels and then let them participate more to the clock signal. The phase modulation

Further author information: (Send correspondence to Alexandre Bouvier)

E-mail: alexandre.bouvier@obspm.fr 
allows the constructive superposition of the CPT dark states [4]. Therefore, the DM scheme allows us to have a good contrast of the atomic transition we use to lock the oscillator on.

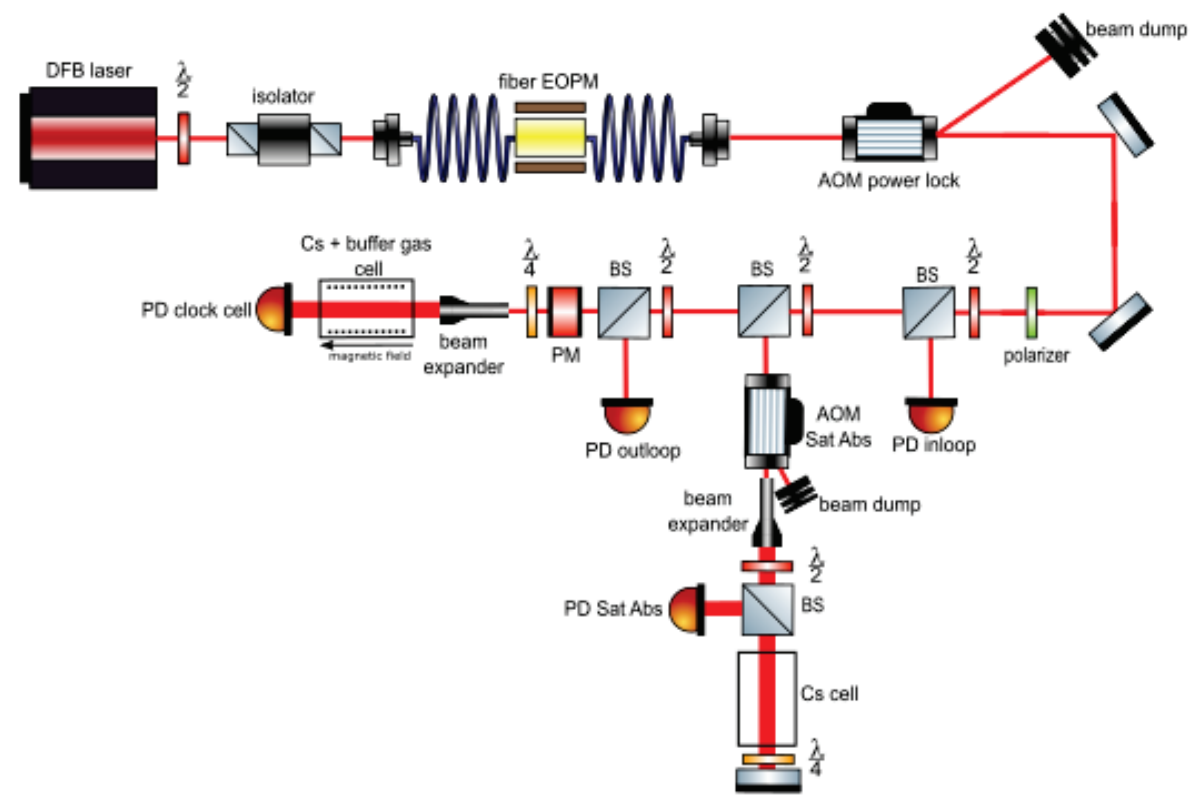

Figure 1. Experimental setup of the DM-CPT atomic clock.

\section{NOISE BUDGET AND STABILITY}

The polarisation and phase are modulated synchronously according to the time sequence depicted in figure 2 and said previously,. At each phase/polarisation, after a time $t_{p}$ where the atoms are pumped in the CPT state, we detect the signal during a time $t_{w}$.
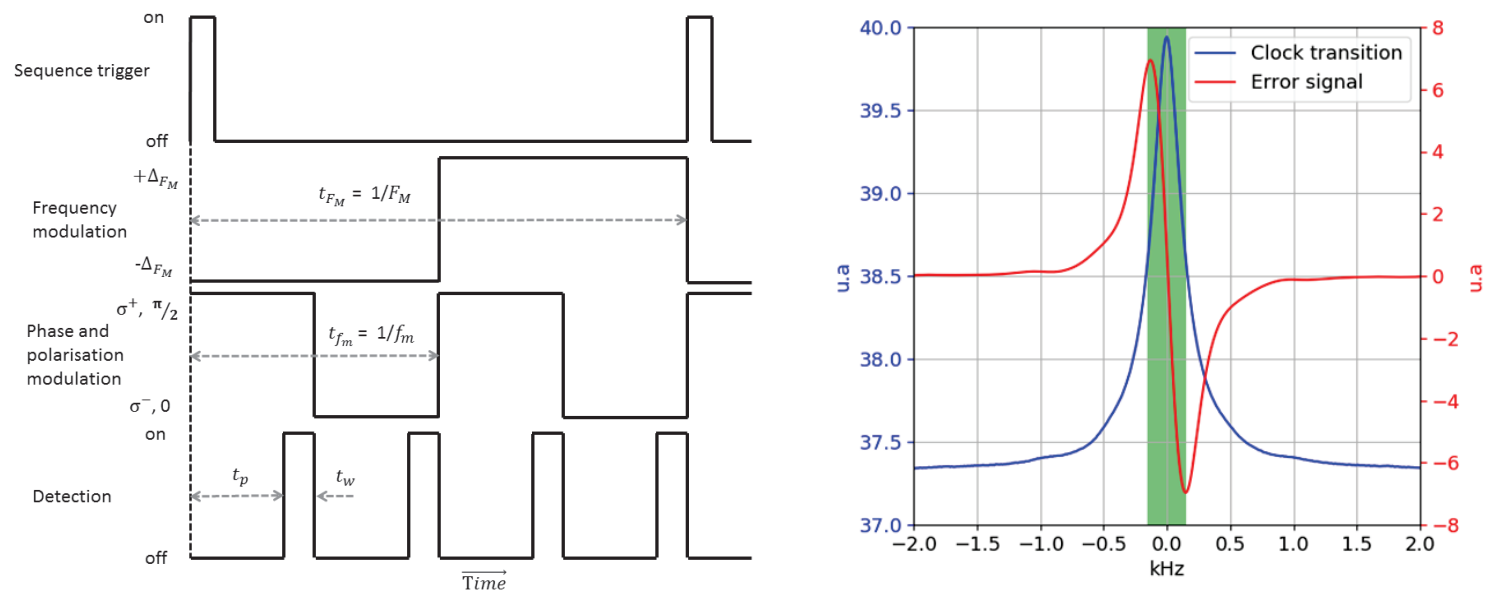

Figure 2. left Time sequence used. Frequency is modulated at $F_{m}=250 \mathrm{~Hz}$ at $\Delta_{F_{M}}=130 \mathrm{~Hz}$ depth. Pumping time and detection window are noted respectively $t_{p}$ and $t_{w}$. right Atomic signal (blue) and error signal (red), we obtained a contrast of $6.98 \%$ for a full width at half maximum of $290 \mathrm{~Hz}$ represented in green.

The error signal used to lock the clock frequency is generated by square modulating the microwave frequency at a frequency of $F_{m}=250 \mathrm{~Hz}$ with a depth of $\Delta_{F_{M}}=130 \mathrm{~Hz}$ around the clock frequency. The obtained signal is also shown in the figure 2, it presents a contrast of $6.98 \%$ for a $290 \mathrm{~Hz}$ at full width at half maximum. 


\begin{tabular}{|c||c|c|}
\hline Noise source & Noise level & Contribution $\sigma_{y}(1 \mathrm{~s})$ \\
\hline \hline Shot noise & $4.85 \mathrm{nV} \cdot \mathrm{Hz}^{-\frac{1}{2}}$ & $3.9 \times 10^{-14}$ \\
\hline Detector noise & $15.9 \mathrm{nV} \cdot \mathrm{Hz}^{-\frac{1}{2}}$ & $1.5 \times 10^{-14}$ \\
\hline Laser AM-AM & $28.2 \mathrm{nV} \cdot \mathrm{Hz}^{-\frac{1}{2}}$ & $1.8 \times 10^{-14}$ \\
\hline Laser FM-AM & $96.0 \mathrm{nV} \cdot \mathrm{Hz}^{-\frac{1}{2}}$ & $4.2 \times 10^{-14}$ \\
\hline Local oscillator & $-118 \mathrm{dBrad}^{2} \cdot \mathrm{Hz}^{-1}$ & $3.4 \times 10^{-14}$ \\
\hline RF power & $3.2 \times 10^{-4} \mathrm{dBm}$ & $2.7 \times 10^{-13}$ \\
\hline \hline Total & & $2.8 \times 10^{-13}$ \\
\hline
\end{tabular}

Table 1. Budget of the contributions to the clock frequency stability at $1 \mathrm{~s}$

Now we will discuss about the main source noises that limit the frequency stability at $1 \mathrm{~s}$ and $1000 \mathrm{~s}$. First, for the short-term stability limitations, we consider only white noise sources and therefore assume that the different contributions can be independently added. The Allan variance can be expressed as the sum of the different contributions:

$$
\sigma_{y}^{2}(\tau)=\sum_{i} \sigma_{y, i}^{2}(\tau)
$$

where $\sigma_{y}^{2}$ and $\sigma_{y, i}^{2}$ are respectively the Allan variances of the clock frequency and the contribution to the clock frequency by the fluctuations of a parameter $i$. When this parameter modifies the clock frequency during an interrogating cycle, we can write $\sigma_{y, i}^{2}$ as:

$$
\sigma_{y, i}^{2}(\tau)=\frac{1}{f_{c}^{2}}\left(\sigma_{i}^{2}\right)_{t_{w}} C_{i, f_{c}}^{2} \frac{T c}{\tau} .
$$

Here, $f_{c}$ is the clock frequency, $\left(\sigma_{i}^{2}\right)_{t_{w}}$ the Allan variance of the parameter $i$ sampled during the detection time $t_{w}, C_{i, f_{c}}=\frac{d f_{c}}{d i}$ is the sensibility of the frequency clock to fluctuations of $i$ and $T_{c}$ is the cycle time of the sequence. It appears clearly that we can measure the sensibility of the clock frequency to a parameter by making significant variations of $i$ and then measuring the effects on $f_{c}$. If we consider that $\left(\sigma_{i}^{2}\right)_{t_{w}} \approx \frac{S_{i}\left(F_{M}\right)}{2 t_{w}}[5], S_{i}\left(F_{M}\right)$ being the power spectral density (PSD) of the i parameter, then we can write:

$$
\sigma_{y, i}^{2}(\tau)=\frac{1}{f_{c}^{2}} \frac{S_{i}\left(F_{M}\right)}{2 t w} \frac{C_{i, V_{c}}^{2}}{S_{l}^{2}} \frac{T c}{\tau}
$$

with $C_{i, V_{c}}=\frac{d V_{c}}{d i}$, the sensibility of the measured signal after the clock cell to a parameter $i$ and $S_{l}$ the slope of the error signal in $\mathrm{V} \cdot \mathrm{Hz}^{-1}$.

For the short-term stability, results are reported in the table 1 . We mainly used the equation explicited in (3) and (1), since it is easier to get the PSD for certain parameters such as the shot noise. We can clearly see that the main contribution is the variations of the microwave power. It is due to the fact that the microwave power influence the balance between the side bands and the carrier, influencing directly the CPT signal. However, the 
sum of the contribution is given to be $2.8 \times 10^{-13}$ at $1 \mathrm{~s}$ which is in good agreement with the measured stability of $2.9 \times 10^{-13} \tau^{-\frac{1}{2}}$ in the figure 3 .

\begin{tabular}{|c||c|c|}
\hline Noise source & Coefficient & Contribution $\sigma_{y}(1000 \mathrm{~s})$ \\
\hline \hline Laser power & $16.1 \mathrm{~Hz} \cdot \mathrm{mW}^{-1}$ & $1.8 \times 10^{-15}$ \\
\hline Laser Frequency & $21.1 \mathrm{mHz} \cdot \mathrm{MHz}^{-1}$ & $6.7 \times 10^{-14}$ \\
\hline Magnetic field & $0.29 \mathrm{~Hz} \cdot \mathrm{pT}^{-1}$ & $4.0 \times 10^{-15}$ \\
\hline Cell temperature & $0.25 \mathrm{~Hz} \cdot \mathrm{K}^{-1}$ & $7.8 \times 10^{-15}$ \\
\hline RF power & $-7.9 \mathrm{~Hz} \cdot \mathrm{dBm}^{-1}$ & $7.6 \times 10^{-13}$ \\
\hline \hline Total & & $7.7 \times 10^{-13}$ \\
\hline
\end{tabular}

Table 2. Budget of the contributions to the clock frequency stability at $1000 \mathrm{~s}$

Concerning the noise contributions at $1000 \mathrm{~s}$, we will refer to the equations (2) and (1). The results are consigned in the table 2. The main limit is still the microwave power, meaning that if we want to gain in stability, we should first focus our effort on it. The reported sum of the contribution noted in the table 2 is $7.7 \times 10^{-13}$, which is not in perfect agreement with the measured stability of $1.1 \times 10^{-12}$ in the figure 3 . We attribute this difference to thermal effects, not fully characterized yet. We suspect back-reflections in electrooptic amplitude modulator (EOAM, pm on the figure 1) that depends on the temperature of the crystal, so it is hard to give even a rough estimation yet because the phenomenon is not linear. However, this matter will soon be studied and hopefully corrected. According to the true nature of the problem, many solutions can be envisaged, such as regulating the temperature of the EOAM or switching to an other device that will not present the same characteristics regarding the temperature. Moreover, we can see on the green curve that the Allan deviation of the clock frequency increases as $\sqrt{\tau}$, characteristic of a random-walk frequency noise. This means that there is some parameters varying independently, such as the microwave power and the EOAM temperature.

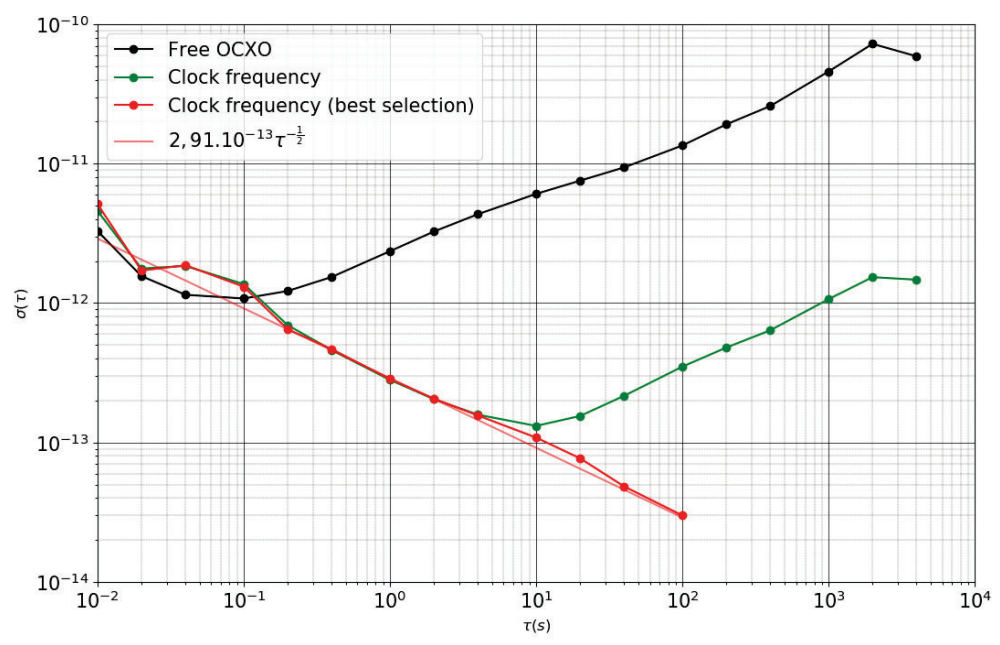

Figure 3. Frequency stability of the DM-CPT. In black, the local oscillator (OCXO) in free running, green locked and red is the best selection with the associated slope in a lighter trait. 


\section{LIGHT SHIFT CORRECTION}

In reality, the excited state can be shifted because of the laser power transmitted on the atoms (AC Stark effect). It results to a shift of the atomic signal as seen on the figure 4. A light shift correction method for the continuous vapor cell atomic clocks has already been published [6,7]. It acts directly on the local oscillator in order to compensate the light shift.
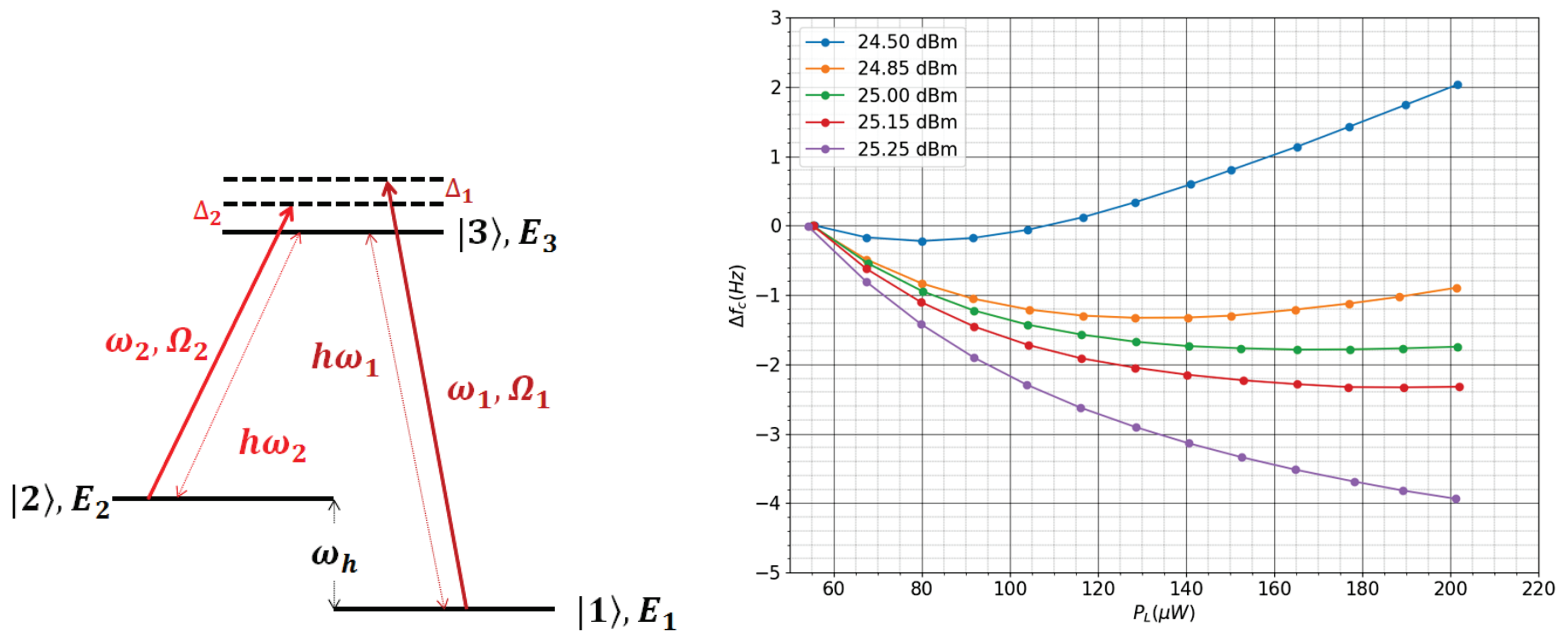

Figure 4. left Illustration of the shifted excited state due to the AC Stark effect. right Clock frequency shift as function of different laser power for different microwave power.

Previous results points out however we need to act on the microwave power in order ameliorate the long term stability of the DM-CPT clock. In our case, the AC Stark effect depends of the microwave power. According to it, the light shift will have a different sign and absolute value as shown in the figure 4. Our idea is to use this propriety by modulating the laser power before the clock cell, in order to generate a error signal to correct the microwave power. By doing so, we hope to compensate the microwave drift, reduce the laser power and frequency contribution. This consideration leads to the new sequence reported in the figure 5 below:

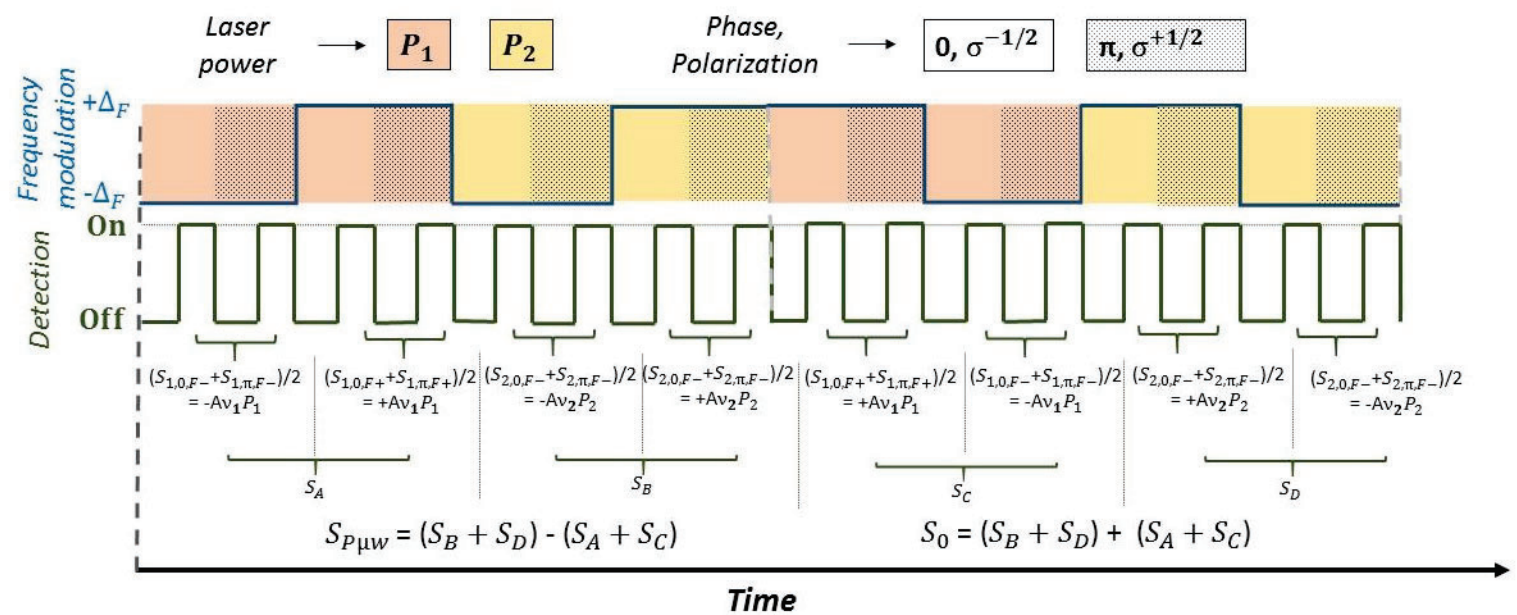

Figure 5. Time sequence used for the microwave correction.

Working at two different laser power can degrade the short term stability. As a results, for a given microwave power, according to the laser power, you obtain a different stability at $1 \mathrm{~s}$ averaging time. As presented in 

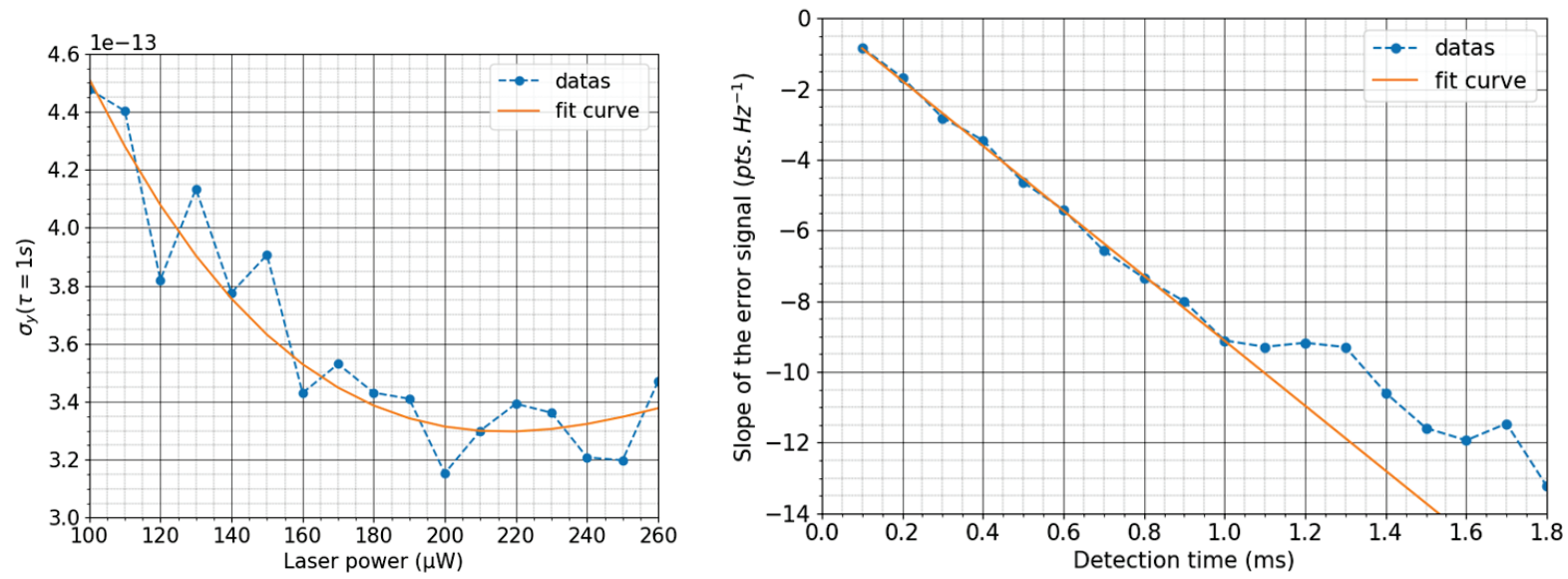

Figure 6. left Stabilities at $1 \mathrm{~s}$ of the DM-CPT atomic clock for various laser powers. right Slope of the error signal as function of the detection time.

the figure 6. Moreover, laser power influence the atomic signal we lock on and so change the error signal, and therefore, the stability of the clock frequency. One way to avoid the degradation is to act on the detection time. The figure 6 shows that by reducing the detection time one can act on the slope of the error signal. Following these results, we chose to set our laser powers to $P_{1}=187 \mu \mathrm{W}$ and $P_{2}=250 \mu \mathrm{W}$ in order to have the best stability at $1 \mathrm{~s}$ and still two laser powers separated enough to generate a correct error signal for the microwave power correction. We then chose to have detection time $t_{w}$ for $P_{1}$ of $0.3 \mathrm{~ms}$ and of $0.5 \mathrm{~ms}$ for $P_{2}$, in order to have respectively an error signal with a slope of $-6.91 \mathrm{pts} . \mathrm{Hz}^{-1}$ and $-6.49 \mathrm{pts} . \mathrm{Hz}^{-1}$.

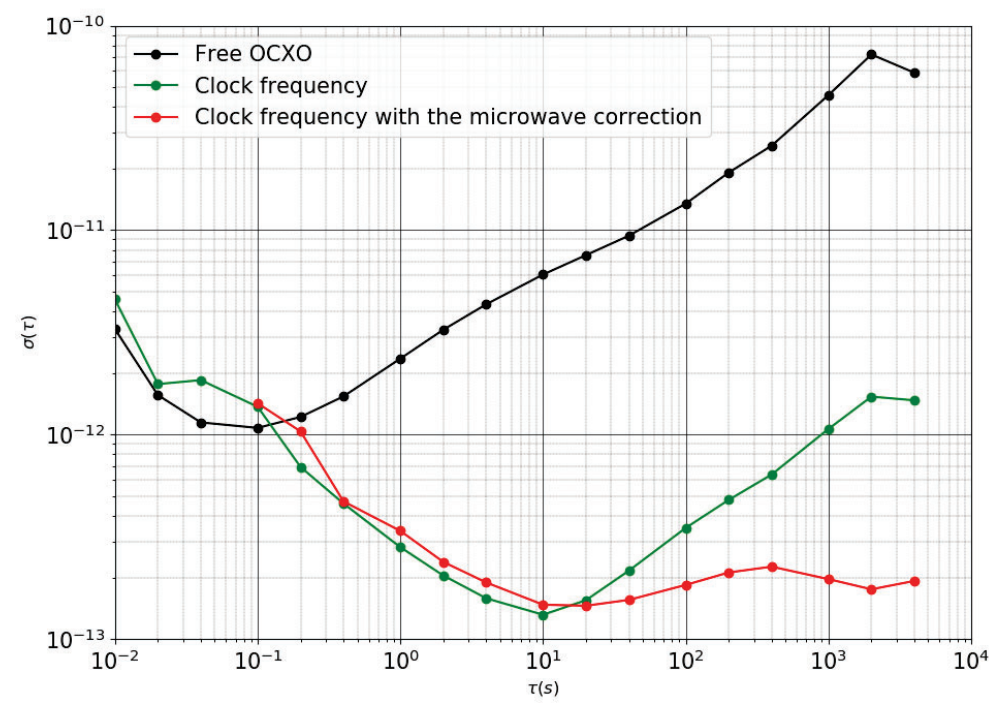

Figure 7. Frequency stability comparison between the OCXO in free running (black), locked with the previous method (green) and with the new microwave locking method (red).

In the figure 7 , we can see that the short term is degraded to $3.3 \times 10^{13}$ at $1 \mathrm{~s}$. This was expected because of the different microwave power we are working with, compared to the results without the microwave power correction, and the results are in agreement with those in the figure 6. We measure a frequency stability of $2.1^{13}$ at $1000 \mathrm{~s}$ which is better by almost a factor 10. The bump around $400 \mathrm{~s}$ lets us think that the new limitation is 
definitely of thermal kind, as stated in the previous section. We will investigate it in order to get rid of it and hopefully go in the $10^{-14}$ region from 20 seconds integration time until $1000 \mathrm{~s}$.

\section{CONCLUSION}

In conclusion, we presented the latest developments on the DM-CPT compact atomic clock. First, we demonstrated stabilities of at $2.9 \times 10^{-13}$ at $1 \mathrm{~s}$ and $1.1 \times 10^{-12}$ at $1000 \mathrm{~s}$, showing the main limits were the microwave power variations. Then, with a a new time sequence and microwave locking method, we demonstrated encouraging results of at $3.3 \times 10^{-13}$ at $1 \mathrm{~s}$ and $2.0 \times 10^{-13}$ at $1000 \mathrm{~s}$. Still, there is some work to do such as investigating on the thermal limitation, or the best working points $\left(\frac{P_{2}}{P_{1}}\right.$, detection times,...). However, the simple and compact optical design of the DM-CPT makes it a good candidate for embarked atomic clocks. The fact that it is a continuous and in line construction is very appealing for satellites clocks or telecommunication and industrial applications.

\section{ACKNOWLEDGMENTS}

We want to thank W. Moreno and L. Absil for their reviews, the electronic department at the SYRTE for their time and precious advices and the European Space Agency for the loan of the Symmetricom 5125a. We also want to thank FIRST-TF for the co-funding of the PhD thesis and the CNES for having followed this project.

\section{REFERENCES}

[1] Esnault, F., Rossetto, N., Holleville, D., Delporte, J., and Dimarcq, N., "Horace: a compact cold atom clock for galileo," Advances in Space Research 47(5), 854-858 (2011).

[2] Langlois, M., De Sarlo, L., Holleville, D., Dimarcq, N., Schaff, J.-F., and Bernon, S., "Compact cold-atom clock for onboard timebase: Tests in reduced gravity," Physical Review Applied 10(6), 064007 (2018).

[3] Yun, P., Tricot, F. m. c., Calosso, C. E., Micalizio, S., François, B., Boudot, R., Guérandel, S., and de Clercq, E., "High-performance coherent population trapping clock with polarization modulation," Phys. Rev. Applied 7, 014018 (Jan 2017).

[4] Yun, P., Guérandel, S., and De Clercq, E., "Coherent population trapping with polarization modulation," Journal of Applied Physics 119(24), 244502 (2016).

[5] Rubiola, E., "Enrico's chart of phase noise and two-sample variances," rubiola.org/pdf-static/Enrico's-chartEFTS.pdf .

[6] Yudin, V., Basalaev, M. Y., Taichenachev, A., Pollock, J., Newman, Z., Shuker, M., Hansen, A., Hummon, M., Boudot, R., Donley, E., and et al., "General methods for suppressing the light shift in atomic clocks using power modulation," Physical Review Applied 14 (Aug 2020).

[7] Abdel Hafiz, M., Vicarini, R., Passilly, N., Calosso, C., Maurice, V., Pollock, J., Taichenachev, A., Yudin, V., Kitching, J., and Boudot, R., "Protocol for light-shift compensation in a continuous-wave microcell atomic clock," Phys. Rev. Applied 14, 034015 (Sep 2020). 